\title{
Web-based multi-criteria decision support system in screening and selection of priority projects for inclusion in agency's annual budget proposal
}

\author{
Taniegra Sussie Tarrega* \\ Polytechnic University of the Philippines, Sta. Mesa, Manila, Philippines
}

\author{
Keywords \\ Data mining methodology \\ Private coach hires \\ Transportation \\ SMEs \\ Data mining application \\ CRISP-DM
}

Received: 3 December 2018

Accepted: 7 January 2019

Published: 20 February 2019

\begin{abstract}
This paper presents a developed web-based decision support system that implements a multi-criteria decision analysis, the Analytic Network Process (ANP) as a modeling tool in screening and selecting an agency's priority projects for inclusion in the annual budget proposal. Screening and selecting priority projects for inclusion in the annual budget proposal of the government is complex decision-making that involves many stakeholders with conflicting priorities and dynamic preferences and limited technical information. Government decision-making becomes more complicated because of the complexities carried by the people involved in the process and other factors contributing to balancing political, technical, and economic considerations. The application of Multi-Criteria Decision Analysis (MCDA) methods for decision-makers helps them facilitate the systematic treatment of information, inform those involved in the process, and develop effective decisions in complex circumstances. The development of a decision support system that implements MCDA tools such as Analytic Network Process (ANP) modeling techniques in screening and selecting priority projects helps manage this complexity and makes decision-making efficient, effective, and transparent. The study used a pre-defined set of criteria identified during the progress of the study.
\end{abstract}

(C) 2019 The Author(s). Published by TAF Publishing.

\section{PROBLEM STATEMENT}

The main objective of the study is to develop a "Web-Based Multi-Criteria Decision Support System (MCDSS) in Screening and Selection of Priority Projects for Inclusion in the Agency's Budget Proposal". To make this feasible, this study sought answers to the following problems:

1. What are the challenges encountered by the respondents in screening and selection of priority projects?

2. What is the level of acceptance by the respondents on the developed "Web-based MCDSS in Screening and Selection of Priority Projects for Inclusion in the Agency's Annual Budget Proposal" in terms of:

2.1 Functionality;

2.2 Reliability;

2.3 User-friendliness; and

2.4 Efficiency;
3. What are the recommendations for the possible improvements of the developed "Web-based MCDSS in Screening and Selection of Priority Projects for Inclusion in the Agency's Annual Budget Proposal"?

\section{SIGNIFICANCE OF THE STUDY}

This study is deemed significant to the following:

\section{A. Government Planners}

This study will benefit government planners in promoting transparency in priority-setting activities of government projects. Implementing standard set of criteria in screening and selection of priority projects will gain the trust and confidence of other stakeholders. It will serve as a guiding tool in deciding which projects among the list are to be pri-

\footnotetext{
${ }^{*}$ Corresponding author: Taniegra Sussie Tarrega

†email: sussie.taniegra@nia.gov.ph
} 
oritized. Moreover, this study will be helpful to the agency's planners in formulating other models of priority strategy.

\section{B. Facilitator}

This study will benefit the facilitators in planning and budget workshop. The output of the system will help and guide them in finalizing the list of projects to be submitted for annual budget proposal $[1,2,3,4]$.

\section{Top Management}

This study will help top managers in viewing the priority projects to be included in the agency's annual budget proposal. Future Researchers. This study may provide future researchers basis for another study by using the methods used in this paper in screening and selection of priority projects in other problem sectors [5, 6, 7].

\section{BACKGROUND OF THE STUDY}

All levels of government have budgets that show how much revenue the government expects to receive in taxes and other income, and how the government plans to spend it. In the Philippines, the Department of Budget and Management (DBM) issues a "Budget Call" every year to officially begin the annual budgeting process of the national government $[8,9]$. This contains guidelines, procedures, and timetables for all government agencies in formulating their budget proposals for the coming year. This requires the agencies to prepare their budgets in accordance with the said guidelines, macro-economic assumption, and ceilings.

The annual government budgeting is in accordance with the Constitution. It also follows the principle that all government spending be justified anew each year. This ensures that the government continuously evaluates and reviews the allocation of resources for cost efficiency and effectiveness. It is therefore of vital importance to all government agency's planner to formulate budget proposal efficiently, effectively, and transparently. Because of this, government's planners must have a systematic and transparent approach of screening and selection of priority projects for inclusion in the annual budget proposal.

Screening and selection of government priority projects for inclusion in the agency's annual budget is a complex decision-making because of the multiple selection of alternatives, several conflicting criteria, and involvement of multiple stakeholders. Balancing between political, technical, and economic considerations makes decision for government planners even more complicated. Pressure from people and administrative requirements necessitate a traceable and transparent method of decision-making.
It is becoming increasingly important for government planners to adopt a multi-criteria decision-making method in screening and selection of priority projects and programs from the long list of projects included in the agency's Investment Plans to allocate limited resources efficiently, effectively and transparently.

The MCDA methods have been developed to support the decision-makers in decision problem generated by the con flicting criteria and complex circumstances $[10,11]$. It also facilitates systematic treatment of the information and factors necessary to make informed decisions. MCDA methods provide stepping-stones and technique for finding a compromise solution. They are not automatable methods that lead to the same solution for every decision maker, but they incorporate preference information that being provided by decision maker, which leads to the compromise solution $[12,13,14]$.

There are several approaches in applying MCDA and its application is even wider as technology evolves to allow complex problems to be addressed with relative ease. The MCDA tools have been incorporated into system to create MCDSS. The use of MCDSS as a decision-making tool has seen increasing use even in the government. This study primary aims to develop a web-based MCDSS implementing ANP for problem modeling in screening and selection of agency's priority projects for inclusion in annual budget proposal. This study used a pre-defined set of criteria identified during the progress of the study. This study does not aim to change the way of screening and selection of priority projects, nor it is believed to substitute for any particular procedure used by agency in selecting priority projects, but it aspires to become a helping tool for planners.

This study is looking into the future of integrating other existing systems to minimize manual input of additional information and include options to import data to be used as alternative which are not included in this study.

\section{THEORETICAL AND CONCEPTUAL FRAMEWORK}

\section{A. Theoretical Framework}

This study is anchored on ANP theory. ANP theory is the extension of Analytic Hierarchy Process (AHP), these are tools used in multi-criteria decision analysis. This theory was followed by the researcher in developing MCDSS application. The AHP consists of one goal, some criteria, and alternatives which are arranged in a hierarchy structure where the lower level elements influence higher level elements, as shown in Figure 1. At some point, decision problems cannot be structured in a hierarchy because they involve dependences between the alternatives: dependence that belong 
to the same level, or dependence of higher level elements on lower level elements. This concern motivated Thomas Saaty to develop ANP, which makes possible a natural development of the problem because it does not impose a structure (2001). As Figure 1 shows, a network can be extended in all directions. In order to obtain the priorities to rank al- ternatives in a decision model, the ANP uses pairwise comparisons. A pairwise comparison matrix is formed when comparing a pair or more pairs of elements with respect to a reference element, which remains unchangeable for all the comparisons $[15,16]$.

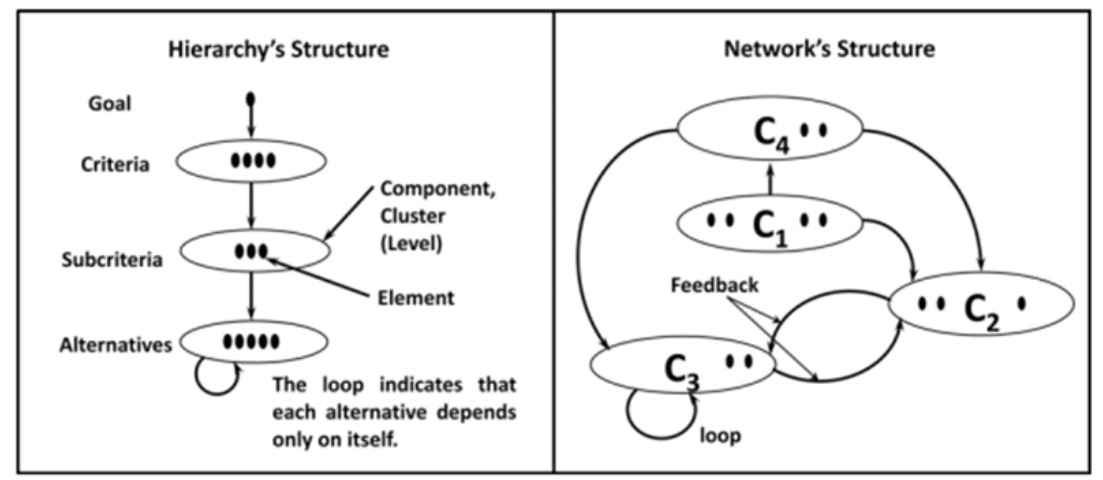

Fig. 1. Hierarchy's and network's structures

To make such comparison, Thomas Saaty developed the scale shown in Table 1, which allows measuring the strength of the judgments (2008).

TABLE 1

SAATY'S FUNDAMENTAL SCALE

\begin{tabular}{lc}
\hline \hline Verbal Judgment & Intensity of Importance \\
\hline Extremely important & 9 \\
Highly more important & 8 \\
Very strongly more important & 7 \\
Very strongly important & 6 \\
Strongly more important & 5 \\
Strongly important & 4 \\
Moderately more important & 3 \\
Moderately important & 2 \\
Equally important & 1 \\
\hline \hline
\end{tabular}

In order to validate the judgments' consistency of the pairwise comparisons matrices, the Consistency Ration (CR) of the matrices is calculated. According to [17], the consistency ratio should be less or equal to 0.1 , where 0.2 is the maximum value that can be tolerated. In order to calculate the consistency ratio of matrix A, for example, it is necessary first to determine the consistency index: Consistency Index (CI) of Matrix A:

$$
C I=\frac{\lambda_{\max }-n}{n-1}
$$

Matrix theory states that a reciprocal matrix, as the case of the pairwise comparison matrix is consistent when the maximum matrix's eigenvalue is equal to the size of a square matrix $n \times n$. In this sense, the consistency index should approach to zero. A pairwise comparison matrix is consistent if their judgments are ruled by the transitivity principal [18]. Before calculating the CR, it is necessary to estimate the averaged Random Consistency Index (RI), which is obtained from random reciprocal matrix using Saaty's fundamental scale. Assuming that a random matrix does not have to be necessarily consistent, it is expected that the RI should be greater than the CI [17], and therefore, the CR should be small. The CR of matrix A is defined as follows:

Consistency Ratio of Matrix A: CR = CI

Random Index (RI)

From the pairwise comparison matrices an eigenvector of priorities will obtained. Those priorities allow comparing the relative importance of some elements respect to other criteria or the element to which they were compared. When all the eigenvector of the decision model are calculated, these are used to form the unweighted supermatrix. To construct this matrix supposes that there are $N$ components. Also, supposes that the component h, denoted by $\mathrm{Ch}, h=1$, $N$, has nh elements, that are denoted by $e h 1, e h 2, \ldots e \square n k$ as shown in Figure 2 [18]. 


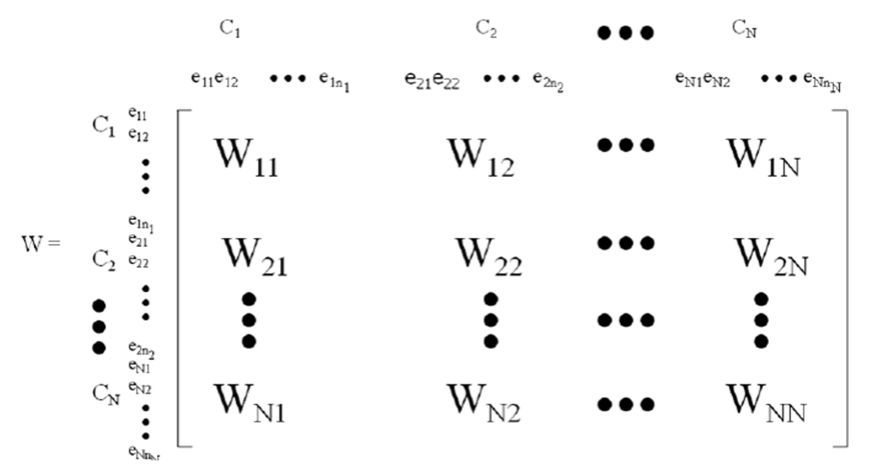

Fig. 2. Supermatrix structure 
In the unweighted supermatrix, each of the eigenvector is assigned to the correspondent column. The elements in the superior row are the criteria used as reference for making the comparison. If there is no influence in the left column element with respect to the criteria in the superior row, the correspondent value in the supermatrix is zero. The next step, according to Saaty methodology, is to construct the weighted supermatrix. The weighted supermatrix comes out from the combination of the unweighted supermatrix and the control hierarchy. The latter scores the priority of a cluster (weight) over all the clusters to which it is connected. All elements in the block corresponding to the intersection between clusters in the unweighted supermatrix will then be multiplied by the weight of the corresponding superior row cluster over its intersection top column cluster, the results will be the weighted supermatrix. This weighted supermatrix was used for ranking alternatives. The approach of ANP that inherits the characteristics of AHP and supports modeling dependencies and feedback between elements in the network makes ANP a more suitable method in selection of priority projects.

\section{B. Conceptual Framework}

Figure 3 represents the conceptual framework of this study using the input, process, and output. The first frame represents the necessary inputs needed to build the web-based MCDSS application. It includes all information encoded in the application such as list of pre-defined decision criteria, list of options of alternatives, and the survey questionnaire. After a series of process cycles, additional inputs are being collected such as tabulated and analyzed responses from selected respondents through interview using survey questionnaires.

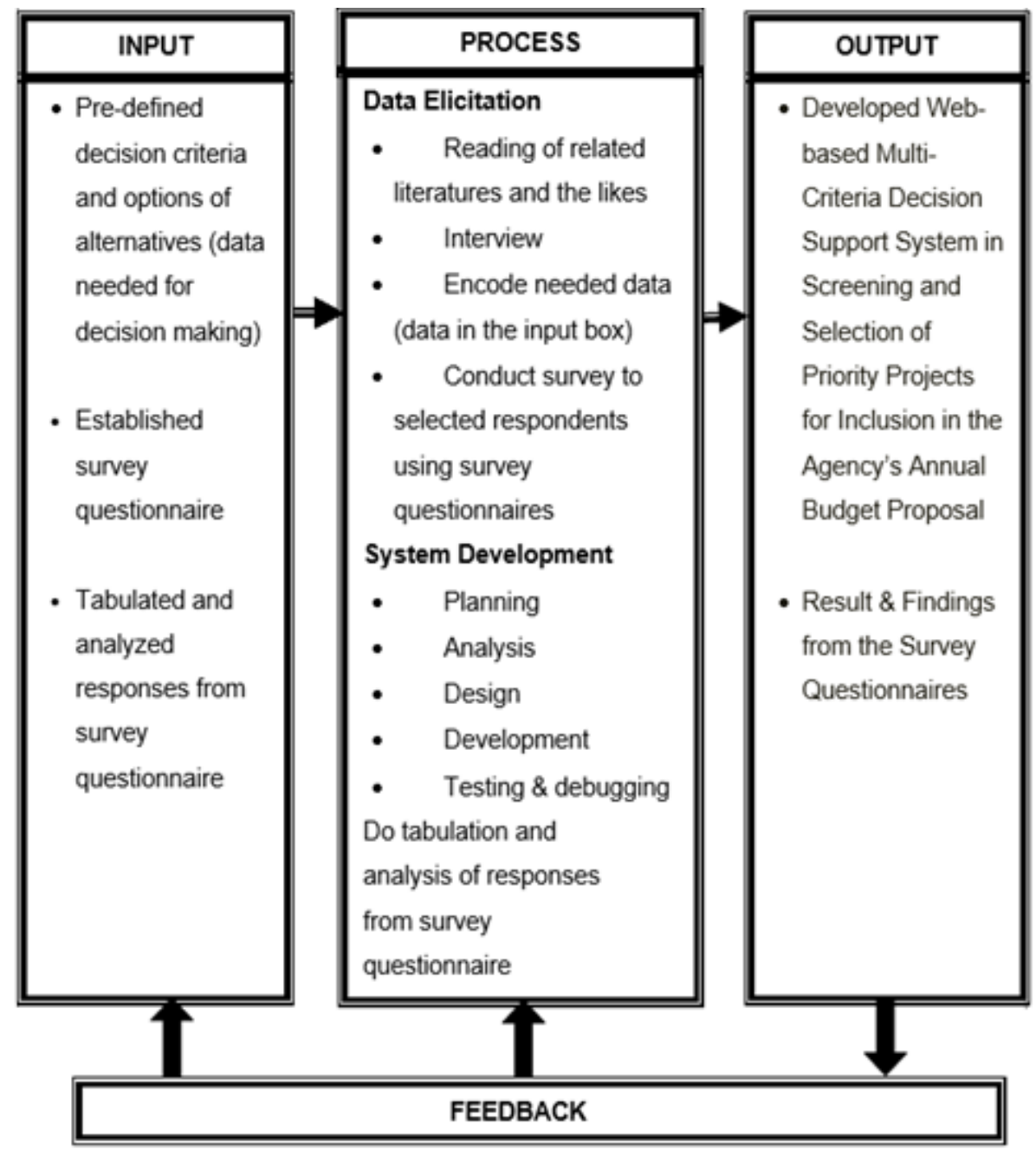

Fig. 3. Conceptual framework of web-based MCDSS application 
The second frame shows the processes needed to be performed to make those identified inputs substantial and meaningful. They include the following activities:

1) Data Elicitation: The researcher starts elicitation by reading related literatures and the likes, interview target respondents, encode/input needed data, establish survey questionnaire, and finally conduct survey to selected respondents using established survey questionnaires.

2) System Development: The researcher followed the System Development Life Cycle (SDLC) such as planning, analysis, design, development, and testing and debugging. Finally, the tabulation and analysis of responses from survey questionnaire were done.

The third frame shows the output of this research which is a developed "Web-based MCDSS in Screening and Selection of Priority Projects for Inclusion in the Agency's Annual Budget Proposal", and the results of survey questionnaire.

\section{Scope and Limitations of the Study}

This study covered individuals involved in the planning and budget preparation of an agency's annual budget proposal. The proposed system is a web-based information system that contains the list of an agency's projects to be used as alternatives, pre-defined set of criteria, list of users with different access privileges, and a pre-defined qualitative scale of importance stored in the database. It has the capability to check an individual's consistency of judgment, auto compute individual's local priority for both criteria and alternatives, and generate ranking results. It has a search function that helps in the retrieval of data. This study was conducted in a government agency, mandated to provide services to farmers nationwide. The researcher demonstrates the developed web-based MCDSS application using eight agency projects or alternatives. These alternatives, including the criteria were judged by the participants where the ranking of alternatives were obtained. It was demonstrated to different agency's planners from central and regional offices that conduct, facilitate, and participate in planning and budget preparation workshop for screening and selection of priority projects for inclusion in an agency's annual budget proposal.

The focus of the study is on the screening and selection of priority projects to be budgeted for the year being proposed. The project that has scheduled allocation for a specific year of implementation specifically the lined projects are not included in this study.

Integrating with agency's existing system to facilitate sharing of data and to minimize manual input are not yet incorporated in the system. Also, the application does not sup- port the importing of data it must be considered in future system enhancements.

\section{METHODS}

The researcher used the descriptive and developmental method of research. Developmental research is defined as "the systematic study of designing, developing and evaluating instructional programs, processes and products that must meet the criteria of internal consistency and effectiveness" [19]. As such, the researcher used this method of research to focus on the design and development of webbased MCDSS application. On the other hand, descriptive method of research was used to identify important factors that could affect the foundation of this study.

For illustrative purposes, the researcher demonstrates the use of develop web-based MCDSS application to different agency's planners from central and regional offices that conduct, facilitate, and participate in planning and budget preparation workshop for screening and selection of priority projects for inclusion in agency's annual budget proposal. The objective of demonstration is to evaluate the features of the developed application in screening and selection of priority projects. The researcher encodes the necessary data for alternatives/options and pre-defined criteria and collects data that corresponds to the objectives of the study through the use of survey. Also, this method addresses "what" questions intended for the target respondents Population, Sample Size, and Sampling Technique. This study intends to solve the research problems through proper collection of necessary data from the target respondents. As identified, the respondents of this study are planners from government agencies responsible in screening and selection of priority projects for inclusion in the agency's annual budget proposal. The target population was chosen because of their actual duties and experience in the above decision problem.

The study drew respondents through random sampling technique and calculate sample size using Cochran's formula. To draw the sample size from the said population, the researcher used Cochran's Formula. This formula as shown below is used to calculate the sample size given the population size and a margin of error:

$$
n=\frac{\frac{z^{2} \times p(1-p)}{e^{2}}}{1+\left(\frac{z^{2} \times p(1-p)}{e^{2} N}\right)}
$$

Where: $n=$ Number of samples

$N=$ Total population

$e=5 \%$ margin of error $(0.05)$

$z=1.96$ for $95 \%$ confidence level 
$p=1$

Using the above formula, out of the 19 total respondents, same number was drawn with $5 \%$ margin of error used as sample size. This margin is quite enough to establish $95 \%$ confidence level on the gathered data.

1) Description of respondent: The 19 respondents who took the survey are agency planners from central and regional offices who conduct, facilitate, and participate in planning and budget preparation workshop for screening and selection of priority projects for inclusion in their agency's annual budget proposal.

2) Research instrument: The researcher used the following instruments in gathering all the data necessary for this study. Each instrument was used to obtain specific data from the target respondents. Available data. The researcher gathered available data on concerned department through its file reports and data published in agency's website.

3) Interview: The researcher conducted interview with the officers responsible in conducting and facilitating planning and budget preparation workshop and personnel of the agency's regional offices also doing the planning and budgeting in their respective regions, to gather specific data needed to establish the foundation of this study.

4) Survey: The researcher made questionnaires that is responsive to this study's statement of the problem. They were distributed to the respondents. Survey allowed the researcher to get the pulse of users by scaling their assessment of the developed web-based MCDSS application. Likert Scale was used to guide the respondents in answering the survey. Typically, Likert scale use different levels shown in Table 2:

TABLE 2

LIKERT SCALE

\begin{tabular}{cll}
\hline \hline Numerical Scale & Rating & Verbal Interpretation \\
\hline 5 & $4.51-5.00$ & Highly Acceptable \\
4 & $3.51-4.50$ & Moderately Acceptable \\
3 & $2.51-3.50$ & Acceptable \\
2 & $1.51-2.50$ & Slightly Acceptable \\
1 & $1.00-1.50$ & Not Acceptable \\
\hline \hline
\end{tabular}

The respondents were given five response options. They were used to quantify their approval/acceptability on the developed application by computing the weighted mean of total responses from all the respondents in every survey questions.

5) Data Gathering Procedure: Readings of related literatures were initially conducted to widen the researcher's view about the topic. This was done through scanning and downloading of different electronic materials available online. They helped the researcher define the specific prob- lems of the study and limit the scope of the study.

Data gathering through interview was conducted to get experts' ideas about the study to be pursued. This allowed the researcher to identify the important factors needed to be considered as well as those data needed to be collected. It also helped establish the technical requirements of the application and define important milestones during its development timeline. For other required data, questionnaires were distributed to gather specific data from target respondents. After which, the data were statistically treated for analysis and interpretation.

\section{A. Data Analysis}

The researcher used Weighted Mean to identify the level of acceptance and approval of the respondents on the developed MCDSS application. Weighted mean is a kind of average where the sum of all score is divided by the number of sources. The formula is shown below:

$$
\mu=\left(\sum X i\right) / N
$$

Where:

$\mu=$ Population Mean

$N=$ Number of Sources

$X i=$ Number of Occurrences

The researcher also used Frequency and Ranking as statistical tools to identify the most to the least problems encountered by the respondents in screening and selection of priority projects.

\section{THE SYSTEM}

\section{A. System Development}

During the system development, the researcher followed the system development life cycle methodology from the start of initial study up to the development of the system and if implemented. The researcher opted to use the opensource development tools such as Java Server Pages (JSP), Apache Tomcat, and MySQL for system development, web server, and database respectively. The open source development tools are found to be advantageous because they are free of cost, open to modification, could be redistributes and with several number of available references.

\section{B. System Architecture}

The developed MCDSS application was intended to use in planning and budget preparation workshop, to be conducted and facilitated by an office responsible in planning and budget preparation of an agency and participated by group of planners from agency's central and regional offices. 


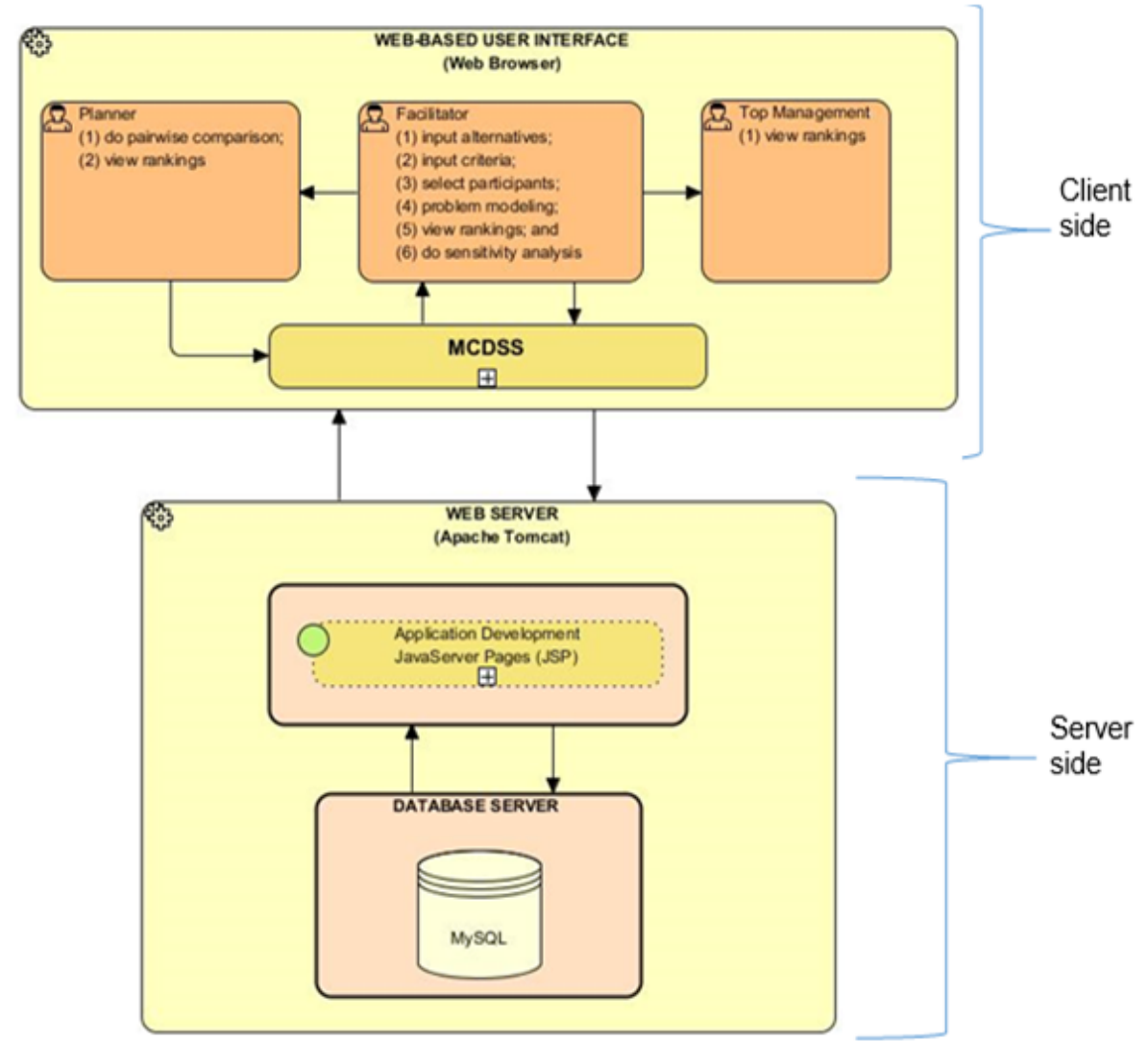

Fig. 4. System architecture of web-based MCDSS application

The developed MCDSS application is a Java web-based interface that runs in Apache Tomcat web server and requires users to use a web browser for encoding, problem modeling and retrieval of data/information from the web server. As shown in Figure 4, the system architecture is divided into two sections: (a) Client side (web-based user interface); and (b) Server side composed of Apache Tomcat web server, JSP for application development, and MySQL as database server.

The client side implements web browser, it communicates with the system's users in order to facilitate user's request then web browser communicates with web server which then interacts with its components JSP and MySQL for data retrieval and management, and sends the results to client side.

The developed MCDSS application has three types of user (a) facilitator; (b) planner; and (c) top management. As shown in Figure 4, the facilitator was in the center of the decision process where most of the activities were lodged. The facilitator guides the group through the relevant stages of the process, carrying out much of the encoding, problem modeling and let the group view ranking results. Before planners could do pairwise comparison, facilitator should let planners participate in the decision process and give them access to the MCDSS application. The facilitator interacts with the system to (a) input alternatives and criteria: for convenience, encoding of alternatives and criteria must be done before the workshop begins; (b) select participants: facilitator will select participants who attended the workshop and will participate in the decision process. The participants have a planner user's account in the system; (c) problem modeling: this is the core function of the system which implements the ANP methodology in screening and selection of priority projects. Problem modeling is only possible if elements (alternatives and criteria) are available in the system, influences, and dependences of this elements must clearly define (d) view rankings: after participants have done their pairwise comparison, the facilitator will let the group view the overall rankings of alternatives; and (e) Finally, the facilitator will do sensitivity analysis.

After the facilitator select participants (planner), the system will notify planners to participate in the decision process. The planner will select which alternatives to be pairwise compare then after selection, system will redirect planner in pairwise comparison of elements (goal, alternatives and criteria). Then the planner will do pairwise comparison of each elements (goal, alternatives and criteria) based 
on their decision's preference considering their knowledge and expertise on the elements being compared. After pairwise comparison, planners can view rankings of alterna- tives which highlight its respective alternatives.

Top management can only view overall ranking of alternatives after planners done all pairwise comparisons.

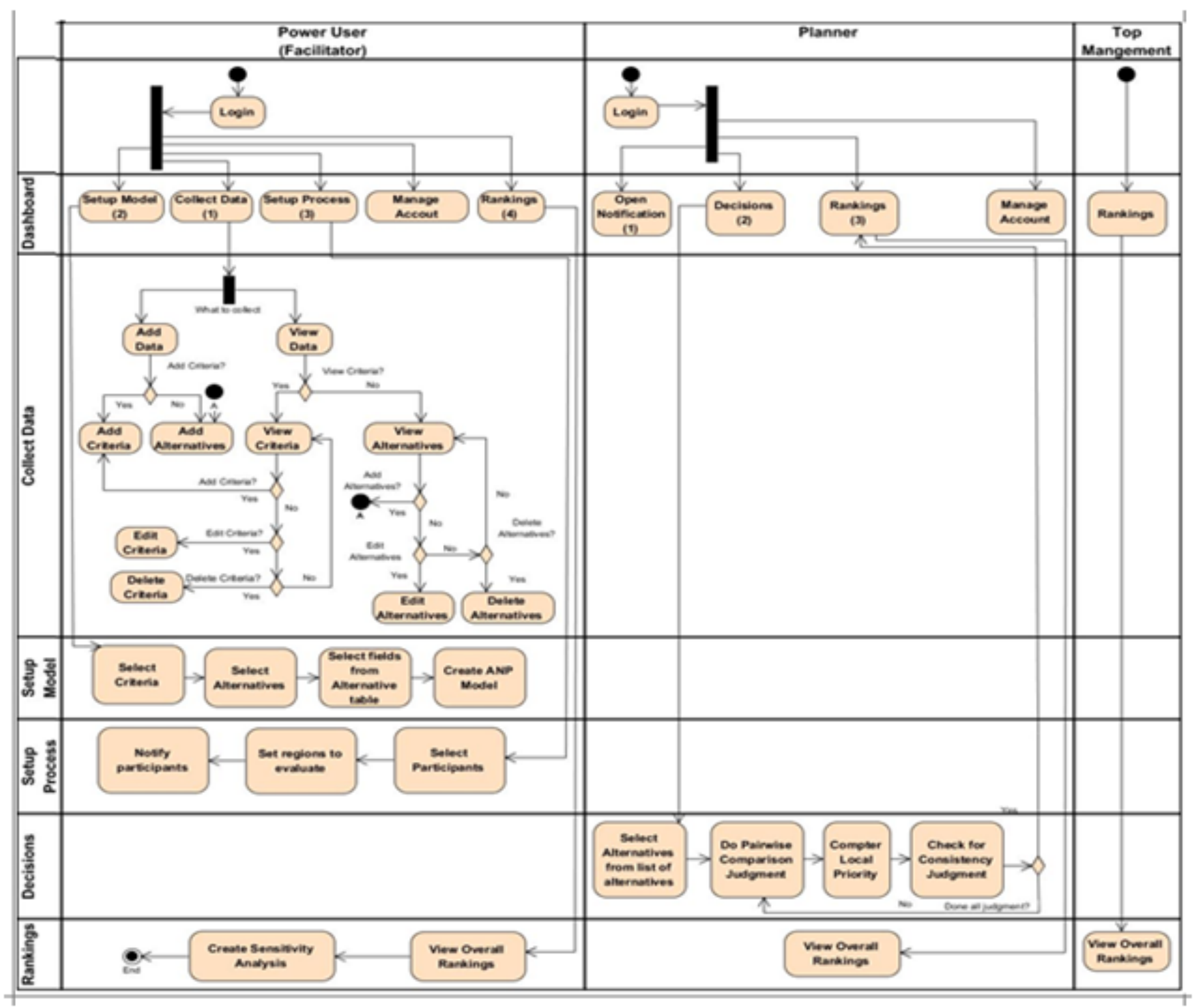

Fig. 5. Activity diagram of web-based MCDSS application

Figure 5 shows the user's individual activity in each module. The Activity Diagram of the developed MCDSS application was shown in Figure 5. It has four (4) columns represented by user and seven (7) rows represented by modules, below discussed how each user interacts with each module:

The developed MCDSS application used a pre-defined set of criteria identified during the progress of the study, this are project accomplishment, actual needs, investment plan, compliance to TRIP and PIP, implementation ready and political interventions.

\section{Facilitator}

The facilitator must login to the developed MCDSS application to access each module in the dashboard. The facilitator in the planning and budget workshop must encode alternatives ("Project Profile") and pre-defined criteria as mentioned above to be used for decision problem ("Select Priority Projects"). Under "Collect Data" module, data for alternatives and, criteria can be viewed, added, edited or deleted. In this paper, eight projects were used as alternative and six criteria. Criteria are grouped into three clus- 
ters according to their relationship: agency criteria (project accomplishment, actual needs, and investment plan), government criteria (compliance to TRIP and PIP and implementation ready), and other factors (political intervention). Encoding of alternatives and criteria must be done before the workshop begins.
To begin with decision process, the facilitator must access the "Setup Process" module to select participants (planner) from the list of users in the developed MCDSS applications to participate in the decision process. The facilitator could set region(s) the participant will evaluate (default is in user's profile), once selected the system will automatically notify selected participants (planner).

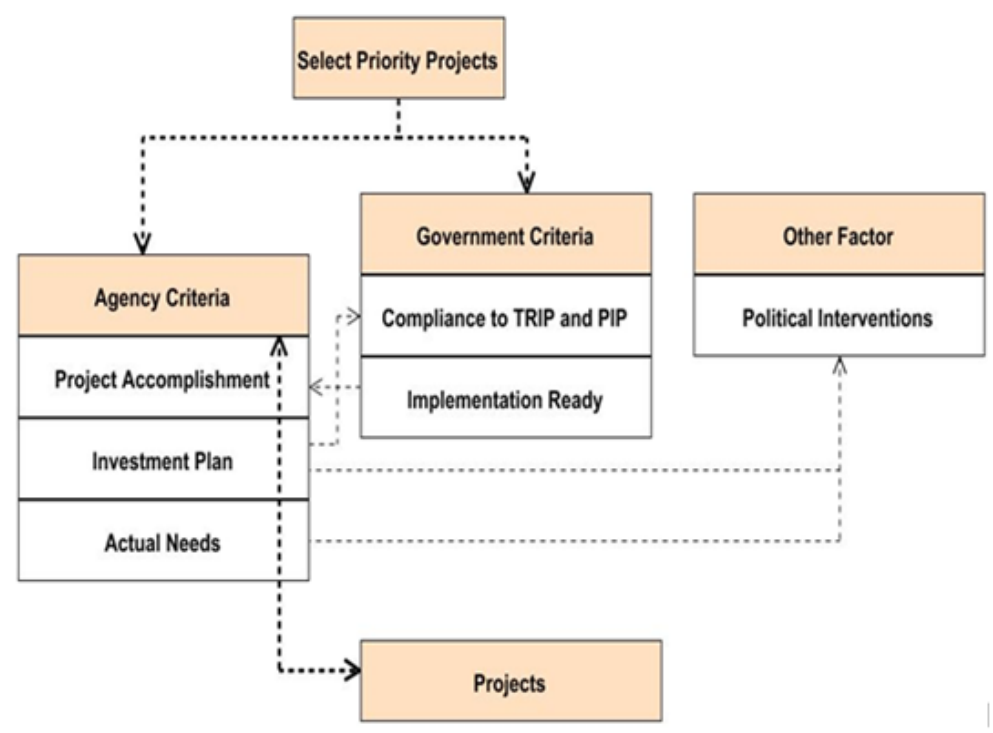

Fig. 6. ANP model structure

After successive interviews with the respondents, and review of the existing literature, this study establishes three levels in the ANP model structure (Figure 6). The heavily dotted line represents direct dependencies of elements while light dotted line represents indirect dependencies. The first level is the decision problem or the Select Priority Projects which is decomposed into two clusters exerting influence into the Select Priority Project", which are Agency Criteria and Government Criteria. Also in this level, the Other Factor cluster has interdependence in Agency Criteria cluster which are further decomposed into three criteria such as: (a) Project Accomplishment; (b) Actual Needs; (c) Investment Plan. Meanwhile, there are two criteria for Government Criteria such as (a) Compliance to TRIP and PIP; and (b) Implementation Ready. Moreover, the one criteria for Other Factor is Political Interventions. As shown in Figure 6, interdependence occurs between the Agency Criteria and Government Criteria, and other interdependence occurs between Agency Criteria and Other Factor.

\section{Setup Model}

While still logged-in, the facilitator must access "Setup Model" module to set-up model structure. Before creating model structure, the facilitator must select criteria (the above created criteria for decision problem: Select Priority Projects), select alternatives (the above pre-defined alternative: "Project Profile"), and select fields from alternative table ("Project").

The direction flow shown in Figure 6 is crucial in ANP problem modeling, it should be consistent to the entire network's structure. This involves directions that indicates dependencies or influences among elements (goal, cluster, criteria, and alternatives). As shown in Figure 6, influences/dependencies are identified during interview to the planners of regional offices. The system will display these influences/dependencies in ANP Model Matrix. Based on this matrix, the system will auto generate questions of pairwise comparison according to the qualitative scale of importance introduced by [17] as shown in Table 2 to be answered by each participants (planner) in "Decisions" module. This ANP model matrix can be viewed in "Tables" module, Model Matrix Tab. Editing of ANP model can no longer be done once participants started to pairwise compare components.

The facilitator monitors the participants' activities through Setup Process module in the Participants list tab and through dashboard. The facilitator's monitoring statuses are as follows: (a) sent: when Facilitator send notification 
to participants; (b) opened; when participant open notification; (c) ongoing; when participants doing the judgments; (d) completed; when judgment completed; (e) unfinished; when participants unable to finish judgment; and (f) closed; when participants unable to participate in the decision process.

After the participants' pairwise comparison judgment was done and all relevant data were computed, the facilitator can now save weighted supermatrix in the Decisions module to let the system compute the overall ranking of alternatives. Finally, the overall ranking of alternatives will be displayed through Rankings module, presented in both graphical and tabular form, it shows which alternative and criteria stand out.

\section{E. Sensitivity Analysis}

The facilitator must check the stability of alternatives ranking to check the results and overall ranking of alternatives obtained through ANP model.

To start with the sensitivity analysis, referring to the results of the above step, the criteria having highest weights are identified by the system. The system will display a slider bar that has fixed set of values for user to use to change criteria's value and compare its effects on the ranking of alternatives. The impact of these weights must be observed on all other alternatives.

\section{F. Planner}

Same as other users, the planner must login to the developed MCDSS application to access each module in the dashboard. When the facilitator sends notification to the planner (participants), a notification appears in the planner's dashboard, Decisions module will only be accessible when notification was opened. After opening notification, the facilitator's monitoring status will change from sent to opened, then the Decisions module will then be accessible. The planner will select alternatives from the list of alternatives (or Project), for demonstration purposes, only eight alternatives among the list will be selected. Number of selected alternatives will be included in the ANP Model Matrix, system will generate new matrix called Overall Model Matrix. This matrix can be viewed in Tables module, Overall Model Matrix tab. After the selection of alternatives, the system will automatically display judgment page for planner to start pairwise comparison judgment, at this point facilitator's participants' status will change from opened to ongoing. Alternatives can be removed from the list as long as pairwise comparison judgment is not yet started.

\section{G. Pairwise Comparison}

Assign weights for each of the elements (criteria and alternatives) to reflect their relative importance to the decision. The elements are weighted according to the qualitative scale of importance introduced by [17] and then it is converted to the quantitative scale range that is from 1 to 9 as shown in Table 1.

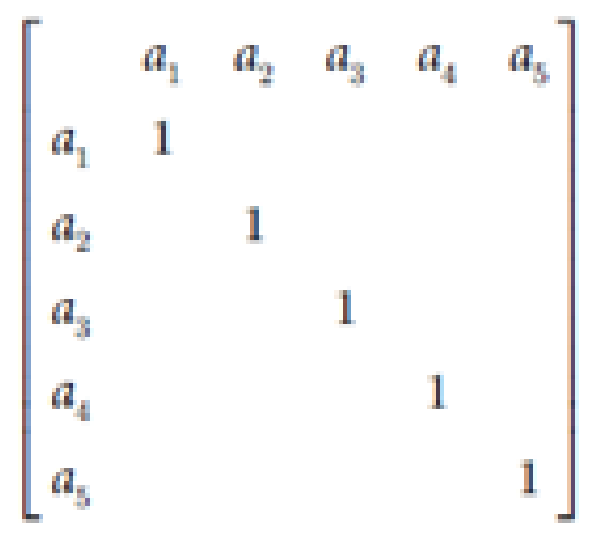

Fig. 7. Pairwise comparison matrix

In the judgment page system will ask planner a series of auto generated questions derived from the ANP Model Matrix (created in the above ANP modeling step). The planner will select judgment from AHP pairwise comparisons in a 1-9 scale to compare criteria with respect to goal and compare for influences to which elements connects based on ANP model. The planner will rate (weight) the list of elements relative to how well they satisfied each interest. The system will display the relative weights of elements in pairwise comparison matrix (Figure 7), where a1, a2, a3, a4, and a5 represent elements, while the diagonal elements having same importance are represented by 1, shown in Figure 7.

\section{H. Calculate Relative Importance}

Weights of the criteria and alternatives are obtained as the elements are normalized. This step has several sub-steps that complete the process of weighting the importance of each criterion and each alternative, and compute the overall ranking of alternatives. 


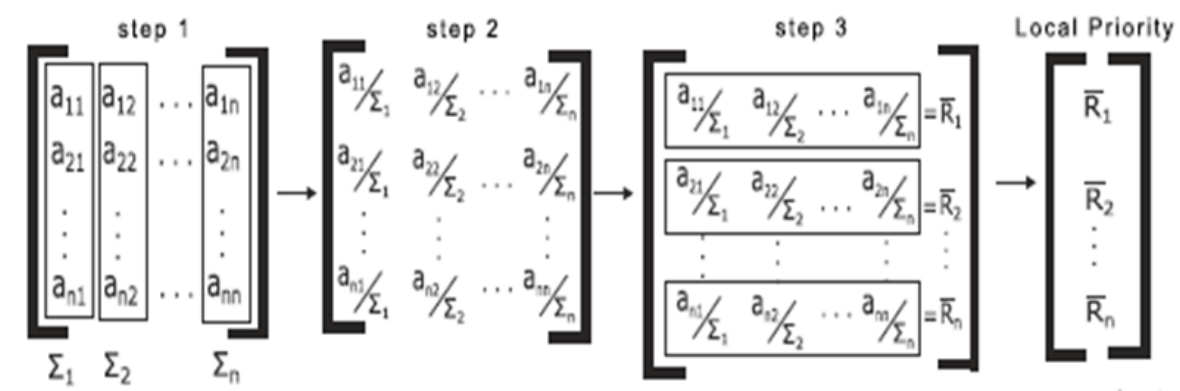

Fig. 8. Calculate local priority

\section{Calculate Local Priority}

From the above inputs (pairwise comparison), the inputs will be processed by the system to generate and display overall priorities for both criteria and alternatives. The system used the a three-step procedure in calculating priorities: (1) sum the values in each column of the comparison matrix; (2) divide each element in a column by the sum of its respective column; and (3) sum the elements in each row of the normalized pairwise comparison matrix, and divide the sum by the $\mathrm{n}$ elements in the row, where $\mathrm{n}$ is the size of the matrix. The result matrix is referred to as the Normalized Matrix.
The results of the above steps are entered in the unweighted supermatrix. This contains the local priorities derived from the pairwise comparisons through the network. The above steps should be done for both criteria and alternative.

\section{J. Check for Consistency}

In order to assure the judgments' reliability, it is very important to ensure consistency between the comparisons made. To check the reliability of pairwise comparisons, the planner must select Consistency Check module. The developed system is adapted from [20] consistency metrics used such as: Consistency Measure (CM), CI, and CR.

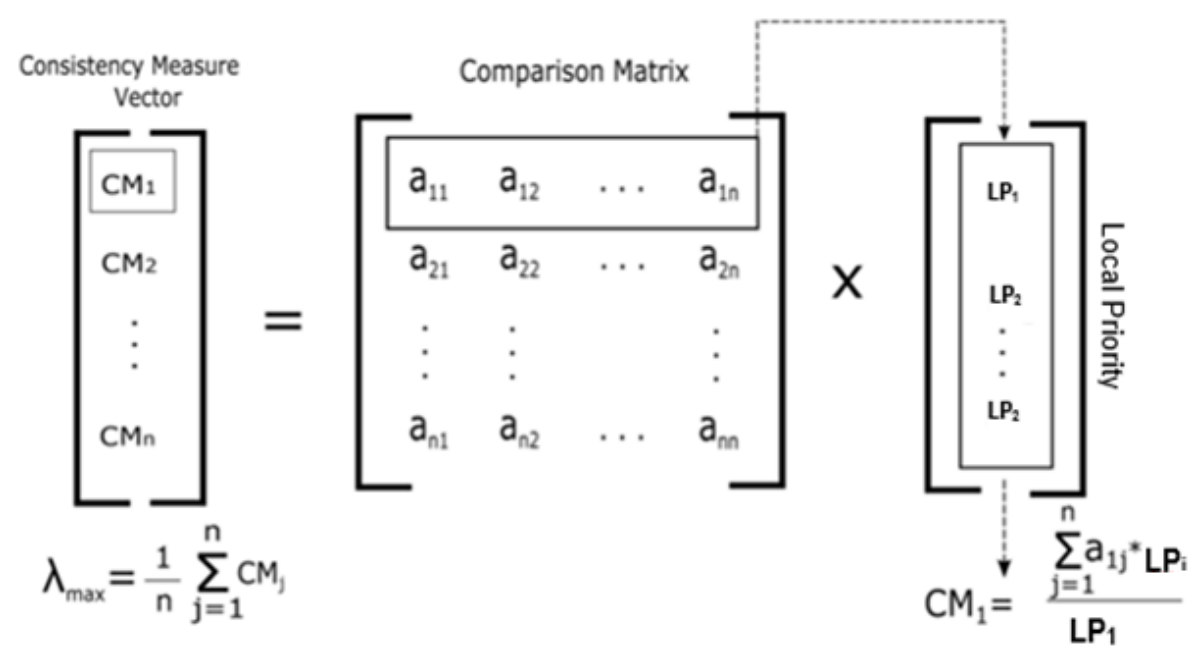

Fig. 9. Calculate consistency measure and maximum priority vector

\section{K. CM}

$\mathrm{CM}$ is the first step in making consistency analysis. The CM is input for consistency index and consistency ratio ( $\mathrm{CI}$ and $\mathrm{CR}$ ) calculation. In order to find CM, Pairwise Comparison Matrix (Figure 9) is first row-wise multiplied with the Local Priority (LP) in Figure 8, and then divided by the corresponding element of the LP as shown in Figure 9.

\section{L. $\quad$ CI}

According to [20], CI is the degree or deviation of consistency. $\mathrm{CI}$ is computed using the following formula:

$$
C I=\frac{\lambda_{\max }-n}{n-1}
$$

Where: $\lambda_{\max }$-is the maximum priority vector, computed as shown in Figure 9.

$n$ - is the size of the matrix.

CR: CR must be calculated using the formula below:

$\mathrm{CR}=\mathrm{CI} / \mathrm{RI}$ 
The acceptable value of $\mathrm{CR}$ is less than or equal to 0.10 , otherwise the pairwise comparison needs to be revised. To get $\mathrm{CR}, \mathrm{CI}$ must be first calculated as shown in the above formula for CI. According to [20], RI is the consistency Index of the random reciprocal matrix generated from the quantitative 9-point scale. The value of RI is shown in Table 3 according to the order of matrix represented by $n$. The value of $n$ in Table 3 was experimentally obtained by [18]. Using the value obtained in the above formula for $\mathrm{CI}$ and the value of RI in a corresponding $\mathrm{n}$ (size of the matrix), CR can now be computed. If the value of $\mathrm{CR}$ is less than or equal to 0.1 , then inconsistency is acceptable, otherwise the pairwise comparison needs to be revised. The remaining matri- ces of all pairwise comparisons need to go through the same process of consistency check or else planner cannot move to the next step. During pairwise comparison judgment, the planner can move back and forth to change their desired preferences. When done all the judgment, planner will then access "Rankings" module to see overall rankings, at this point, facilitator's participant's status will change from "ongoing" to "completed". The developed system will highlight planner's selected alternatives from the list to easily see the ranks of their selected alternatives among the list. Planner can also access "Manage Account" module to edit their personal profile.

TABLE 3

RANDOM INDEX

\begin{tabular}{cccccccccc}
\hline \hline $\mathrm{N}$ & 1 & 2 & 3 & 4 & 5 & 6 & 7 & 8 & 9 \\
\hline $\mathrm{RI}$ & 0 & 0 & 0.52 & 0.89 & 1.11 & 1.25 & 1.35 & 1.40 & 1.45 \\
\hline \hline
\end{tabular}

After the pairwise comparison judgment of all participants and the developed system done computation of the above data, the system will then enter these computed data in matrixes called unweighted supermatrix of individual participants then the system will compute the average mean of each unweighted supermatrix to get the overall unweighted supermatrix of all participants. This overall unweighted and weighted supermatrix is further explain below.

\section{Overall Unweighted and Weighted Supermatrix}

The results of the above step (compute relative importance) are entered in the unweighted supermatrix of the ANP model. It contains the local priorities of the elements obtained from the comparison matrices of each participants (planner). The local priority of each individual matrix is combined which form the unweighted supermatrix. The system will get the average mean of the unweighted supermatrix to get the overall unweighted supermatrix of all participants. This overall unweighted supermatrix is then transformed into weighted supermatrix. Weighted supermatrix is the outcome of overall unweighted supermatrix and cluster matrix. The developed system displays the unweighted and weighted supermatrix in the "Tables" module of participant's dashboard.

\section{N. Top Management}

Top management's dashboard displays the overall ranking of alternatives. To summarize, the process of the developed web-based MCDSS application follows the MCDA step-bystep procedures as follows: (1) establish the goal and decision context; (2) identify criteria; (3) identify the options

ISSN: 2414-4592

DOI: $10.20474 /$ jater-5.1.1 to be appraised; (4) weighting (5) Calculate relative importance; (6) Check for consistency; and (7) sensitivity analysis.

\section{RESULTS}

The following results were obtain, it includes the statistical treatments made on the data, the analysis and interpretation of results using survey questionnaires elicited to the respondents.

Based from the results presented in Table 4, Political interventions ranked 1st in the list of challenges encountered with 3.272 weighted mean. At 2nd place 7.309 weighted mean. The challenges which placed 3rd, 4th, 5th, and 6th have a very closed weighted mean of $12.363,12.618$, 12.872 , and 12.890 respectively for Unachievable targets being presented, Validation time constrains due to late submission, Poor coordination between stakeholders, and Lack of participation of various stakeholders in the decision making for selection of priority projects respectively. Ranked at 7 th is Inadequate time to cope with the agency's policy with 13.309 weighted mean. Meanwhile, Unable to visualize the impact of various selection criteria in the alternative ranking is ranked at 8th with 13.963 weighted mean. Ranked at 9 th with 15.418 weighted mean is Insufficient number of manpower doing the works. Lastly at 10th rank is Uninformed leadership fund with 28.981 weighted mean. From the above results, it can be interpreted that among the list of challenges encountered by the respondents, Political Interventions with the least weighted mean of 3.272 is the most encountered challenge during screening and selection of priority projects. 
TABLE 4

CHALLENGES ENCOUNTERED BY THE RESPONDENTS IN SCREENING AND SELECTION OF PRIORITY PROJECTS

\begin{tabular}{|c|c|c|}
\hline Challenges Encountered & Weighted Mean & Rank \\
\hline 1. Lack of participation of various stakeholders in the decision making for selection of priority projects & 12.890 & 6 \\
\hline 2. Poor coordination between stakeholders & 12.872 & 5 \\
\hline 3. Inadequate time to cope with the agency's policy & 13.309 & 7 \\
\hline 4. Insufficient number of manpower doing the works & 15.418 & 9 \\
\hline 5. Lack of evaluation results in selection of priority projects & 7.309 & 2 \\
\hline 6. Unable to visualize the impact of various selection criteria in the alternative ranking & 13.963 & 8 \\
\hline 7. Unachievable targets being presented & 12.363 & 3 \\
\hline 8. Validation time constrains due to late submission & 12.618 & 4 \\
\hline 9. Political interventions & 3.272 & 1 \\
\hline 10. Uninformed leadership fund & 28.981 & 10 \\
\hline
\end{tabular}

TABLE 5

LEVEL OF ACCEPTANCE OF THE RESPONDENTS ON THE DEVELOPED WEB-BASED MCDSS APPLICATION

\begin{tabular}{|c|c|c|}
\hline Statements & Mean & Interpretation \\
\hline $\begin{array}{l}\text { 1. The developed system provides participation of various stakeholders in the decision making } \\
\text { for selection of priority projects. }\end{array}$ & 3.84 & Moderately Acceptable \\
\hline 2. The developed system can act as communication tool by documenting evaluation results. & 3.79 & Moderately Acceptable \\
\hline 3. The developed system equipped with problem modeling to identify and define selection criteria. & 3.58 & Moderately Acceptable \\
\hline $\begin{array}{l}\text { 4. The developed system performs structured and analytical analysis in screening and selection } \\
\text { of priority projects. }\end{array}$ & 3.58 & Moderately Acceptable \\
\hline 5. The developed system validates and generates evaluation results in time. & 3.37 & Acceptable \\
\hline $\begin{array}{l}\text { 6. The developed system can do sensitivity analysis to show the impacts of modifying one of cri- } \\
\text { teria's values in the ranking of alternative. }\end{array}$ & 3.26 & Acceptable \\
\hline $\begin{array}{l}\text { 7. The developed system ensures public trust and establishing transparency in screening and se- } \\
\text { lection of priority projects. }\end{array}$ & 3.47 & Acceptable \\
\hline 8. The developed system can search and provide desired results. & 3.68 & Moderately Acceptable \\
\hline $\begin{array}{l}\text { 9. The developed system can do consistency checks in user's preference entry and provide sug- } \\
\text { gestion in inconsistent data. }\end{array}$ & 3.58 & Moderately Acceptable \\
\hline Overall Mean & 3.57 & \\
\hline Moderately Acceptable & & \\
\hline
\end{tabular}

Based on the data gathered, the application's functionality is Moderately Acceptable for the users with an overall mean of 3.57. It is also shown in the same table that the users moderately agreed that The developed system provides participation of various stakeholders in the decision making for selection of priority projects, which is one of the objectives of the developed system. This functionality obtained an average mean of 3.84 which is interpreted as Moderately Acceptable, and ranked 1st amongst other functionalities. Ranked 2nd is the statement, System can act as communication tool by documenting evaluation results with an average mean of 3.79, interpreted as Moderately Acceptable. Three of its functionality which are problem modeling, analytical analysis and consistency checks got an average mean of 3.58, and were interpreted as Moderately Acceptable. Meanwhile, search and provide results with an average mean of 3.68 was also interpreted as Moderately Acceptable. Also, another three of its functionality which are system validation, do sensitivity analysis, and ensure public trust and transparency received an average mean of 3.37, 3.26, and 3.47 respectively, which was interpreted as Acceptable.

Table 6 shows the respondents' assessment on the reliability of the developed web-based MCDSS Application. As gathered, the application's reliability received a grand mean of 3.49 which is interpreted as Acceptable. It shows that the reliability of the developed application is deemed acceptable to the respondents. The highest mean of 3.68 or Moderately Acceptable is the reliability result for the model specified dependencies. The consistency of the application on generating model matrix and matrix dependencies obtained a rating of 3.58 or interpreted as Moderately Acceptable. The ability of the system to detect inconsistency of judgment received 3.37 weighted mean or interpreted as Acceptable. Finally, the reliability of doing sensitivity analysis was rated 3.32 or Acceptable. 
TABLE 6

LEVEL OF ACCEPTANCE OF THE RESPONDENTS ON THE DEVELOPED WEB-BASED MCDSS APPLICATION

\begin{tabular}{|c|c|c|}
\hline Statements & Mean & Interpretation \\
\hline 1. The developed system can provide model specified dependencies. & 3.68 & Moderately Acceptable \\
\hline 2. The developed system can provide model matrix consistent with model specified dependencies. & 3.58 & Moderately Acceptable \\
\hline 4. The developed system's sensitivity analysis helps user analyzed results. & 3.32 & Acceptable \\
\hline Overall Mean & 3.49 & \\
\hline Acceptable & & \\
\hline
\end{tabular}

TABLE 7 LEVEL OF ACCEPTANCE OF THE RESPONDENTS ON THE DEVELOPED WEB-BASED MCDSS APPLICATION

\begin{tabular}{lll}
\hline \hline Statements & Mean & Interpretation \\
\hline 1. The developed system can easily navigate and use. & 3.42 & Acceptable \\
2. The developed system graphical user interface and layouts are visually fair. & 3.47 & Acceptable \\
3. The developed system texts and graphical representations are easy to understand. & 3.42 & Acceptable \\
Overall Mean & 3.44 & \\
Acceptable & & \\
\hline \hline
\end{tabular}

Table 7 shows the respondents' assessment on the user friendliness of the developed web-based MCDSS application. As gathered, the application's user friendliness, received 3.44 grand mean which is interpreted as Acceptable. It shows that the developed application was acceptable to the respondents. The highest mean obtained was 3.47 or Acceptable which was found at graphical user interface and layout are visually fair, while the two other options both got an average mean of 3.42 interpreted as Acceptable".

TABLE 8

LEVEL OF ACCEPTANCE OF THE RESPONDENTS ON THE DEVELOPED WEB-BASED MCDSS APPLICATION

\begin{tabular}{lll}
\hline \hline Statements & Mean & Interpretation \\
\hline 1. The developed system behaves in a timely manner. & 3.68 & Moderately Acceptable \\
2. The developed system easily provides and retrieves desired data. & 3.42 & Acceptable \\
3. The developed system provides needed information and view reports as needed. & 3.37 & Acceptable \\
4. The developed system has acceptable response and processing time. & 3.68 & Moderately Acceptable \\
Overall Mean & 3.54 & \\
Moderately Acceptable & & \\
\hline \hline
\end{tabular}

Table 8 shows the respondents' assessment on the efficiency of the developed web-based MCDSS application. As gathered, the application's efficiency, received 3.54 grand mean which is interpreted as Moderately Acceptable. It shows that the developed application is found to be moderately acceptable for the respondents. Specifically, the respondents appreciate the system's timely response and processing time which obtained the highest mean of 3.68 or interpreted as Moderately Acceptable while data retrievals and viewing of reports got an average mean of 3.42 and 3.37 respectively, interpreted as Acceptable. Using the survey results shown and presented in Tables 5-8 for each category, Table 9 shows the overall summary of results.

Table 9 show that the highest level of acceptance from the respondents was obtained with Functionality of the devel oped application with an average mean of 3.57 and is interpreted as Moderately Acceptable. Efficiency received an average mean of 3.54, interpreted as Moderately Acceptable while Reliability got an average of 3.49 or Acceptable while User Friendliness got an average of 3.44 or interpreted as Acceptable. It shows that the overall level of acceptance of the developed application is Moderately Acceptable with a grand mean of 3.51 .

Eleven of the respondents recommended to include in the developed application the importing of alternatives, while eight recommended to include the integration of the developed application to other agency's existing applications. 
Also, other suggestion included the presentation of the developed application in a much longer time to include actual demonstration on pre-selected Irrigation Management Office (IMO) projects.

TABLE 9

RESPONDENT'S LEVEL OF ACCEPTANCE ON THE DEVELOPED WEB-BASED MCDSS APPLICATION

\begin{tabular}{lll}
\hline \hline Parameter & Mean & Interpretation \\
\hline 1) Functionality & 3.57 & Moderately Acceptable \\
2) Reliability & 3.49 & Acceptable \\
3) User Friendliness & 3.44 & Acceptable \\
4) Efficiency & 3.54 & Moderately Acceptable \\
Grand Mean & 3.51 & \\
Moderately Acceptable & & \\
\hline \hline
\end{tabular}

TABLE 10

RECOMMENDATIONS FOR POSSIBLE IMPROVEMENTS OF THE DEVELOPED WEB-BASED MCDSS APPLICATION

\begin{tabular}{ll}
\hline \hline Recommendations & Number of Reponses \\
\hline The developed system should include options to import alternatives & 11 \\
The developed system should include integration to other systems of the agency & 8 \\
Other & 1 \\
\hline \hline
\end{tabular}

\section{FINDINGS}

Based on the data gathered and analysis, the results showed the following:

1. Among the 10 challenges encountered by the respondents in the screening and selection of priority projects, Political Interventions rank first with 3.272 weighted mean which means that the presence of political interventions was observed during the screening and selection of the agency's priority projects. The second most observed challenge is the Lack of evaluation results in selection of priority projects with 7.309 weighted mean, while Uninformed leadership fund is the least encountered challenge with 28.981 weighted mean.

2. The developed web-based MCDSS Application has various features which were assessed in terms of functionality, reliability, user friendliness, and efficiency. With a total grand mean of 3.51, the respondents' level of acceptance of the said features is interpreted as Moderately Acceptable. It shows that the respondents highly approve of the overall design, functionalities, and purpose of the application. In terms of the application's functionality, the respondents rated it with Moderate Acceptance; specifically, the application's setup, judgment and generates decisions, and carry out other features. It received a total mean of 3.57. Efficiency ranked second with a total mean of 3.54 or interpreted as Moderately Acceptable. In terms of Reliability, a total mean of 3.49 or interpreted as Acceptable. It means that the respondents assessed the application's reliability in terms of its consistency in modeling and providing accurate decision results. In terms of user friendliness, a total mean of 3.44 or interpreted as Acceptable was obtained from the responses. The developed application graphical user interface, layouts, navigation, and ease of use are rated acceptable.

3. From the gathered responses, 11 respondents' recommended the inclusion of options to import alternatives on the developed application while eight recommended the integration of the developed application to other agency's existing applications.

\section{CONCLUSION}

Based on the findings, the researcher came up with the following conclusion:

1. Political Intervention is the most encountered challenge by the respondents followed by Lack of evaluation results in selection of priority projects. The said challenges may affect or have impact in the screening and selection of priority projects. Therefore, implementing ANP methods in screening and selection of priority projects that allows influences/dependences like "Political Intervention" that influence one of the criteria would addressed the challenge encountered by the respondents. On the other hand, "Uninformed leadership fund" having been the least encountered challenge is considered with most minimal effect in the screening and selection of priority projects. 
2. The developed Web-based MCDSS application has features and performance which make it moderately acceptable for users. Respondents perceived that the application is functional, reliable, user friendly, and efficient in generating decision results and judgment, ranking of alternatives, and other relevant information necessary for the users. Based on the positive feedback of the respondents, the results generated is concluded as moderately acceptable as this evaluation was done by the respondents who are expert in screening and selection of priority projects that added more confidence in using the application as decision support tool.

3. In the developed web-based MCDSS application, the recommendation of the respondents included future improvements like minimizing the encoding of alternatives.

\section{RECOMMENDATIONS}

The researcher would like to recommend the following based on the findings of the study and conclusions made by the researcher:

1. The developed MCDSS application is a tool that could be used to help facilitators and planners in the screening and selection of priority projects. Through the adaption of the application, the challenges encountered by the respondents may be minimized. The most encountered challenge by the respondents in the screening and selection of priority projects is the "Political Interventions" which is one of the pre-defined criteria provided by the respondents. By considering it as criteria, it lessens the impact in the screening and selection of priority projects. The second chal- lenge encountered by the respondents is the Lack of evaluation results in selection of priority projects which was answered by the developed application through the generation of evaluation as one of the features of the developed application. Similarly, Unable to visualize the impact of various selection criteria in the alternative ranking, another feature of the developed application, intends to visualize the impact of criteria in ranking of alternatives. The developed application could be a means of communication between stakeholders. It will facilitate the coordination and participation of various stakeholders in the decision-making. All stakeholders could be part of the decision-making, provided they are registered in the system.

2. The developed MCDSS application was recommended for use in planning and budget preparation workshop that is to be conducted and facilitated by the office responsible in planning and budget preparation, and participated in by group of planners from agency regional offices. The application's features and performance are efficient in generating decision results and judgment, ranking of alternatives, and other relevant information needed by the planners.

3. For the continuous improvement of the developed application, the researcher will implement the respondents' recommendations for the enhancement of the proposed application. Future researchers may research on other MCDA tools that could be integrated in the developed application to provide comparison of decision results. This will help planners to have other options to be considered in decisionmaking.

\section{REFERENCES}

[1] L. Anojkumar, M. Ilangkumaran, and V. Sasirekha, "Comparative analysis of MCDM methods for pipe material selection in sugar industry," Expert Systems with Applications, vol. 41, no. 6, pp. 2964-2980, 2014. doi: https://doi.org/10.1016/ j.eswa.2013.10.028

[2] K. Babu, P. G. Rajulu, A. R. Reddy, and A. N. Kumari, "Selection of architecture styles using analytic network process for the optimization of software architecture," International Journal of Computer Science and Information Security, vol. 8, no. 1, pp. 45-50, 2010.

[3] G. Buyukozkan and G. Cifci, “A novel hybrid MCDM approach based on fuzzy DEMATEL, fuzzy ANP and fuzzy TOPSIS to evaluate green suppliers," Expert Systems with Applications, vol. 39, no. 3, pp. 3000-3011, 2012. doi: https://doi.org/ 10.1016/j.eswa.2011.08.162

[4] M. Dagdeviren and I. Yuksel, "Personnel selection using analytic network process," Istanbul Ticaret Universitesi Fen Bilimleri Dergisi, vol. 6, no. 11, pp. 99-118, 2007.

[5] T. L. Saaty and D. Ergu, "When is a decision-making method trustworthy? Criteria for evaluating multi-criteria decisionmaking methods," International Journal of Information Technology \& Decision Making, vol. 14, no. 6, pp. 1171-1187, 2015.

[6] F. De Felice, Research and applications of AHP/ANP and MCDA for decision making in manufacturing. New York, NY: Taylor and Francis, 2012.

[7] M. El Amine, J. Pailhes, and N. Perry, “Critical review of multi-criteria decision aid methods in conceptual design phases: 
Application to the development of a solar collector structure," Procedia CIRP, vol. 21, no. 6, pp. 497-502, 2014. doi: https://doi.org/10.1016/j.procir.2014.03.134

[8] E. Gagatsi, G. Giannopoulos, G. Aifantopoulou, and G. Charalampous, "'Stakeholders-based multi-criteria policy analysis in maritime transport: From theory to practice," Transportation Research Procedia, vol. 22, pp. 655-664, 2017. doi: https://doi.org/10.1016/j.trpro.2017.03.062

[9] E. Kujawski, "4.7. 3 multi-criteria decision analysis: Limitations, pitfalls, and practical difficulties," in INCOSE International Symposium, California, CA, 2003.

[10] U. Baizyldayeva, O. Vlasov, A. A. Kuandykov, and T. B. Akhmetov, "Multi-criteria decision support systems: Comparative analysis," Middle-East Journal of Scientific Research, vol. 16, no. 12, pp. 1725-1730, 2013.

[11] A. N. Noorzad and T. Sato, "Multi-criteria fuzzy-based handover decision system for heterogeneous wireless networks," International Journal of Technology and Engineering Studies, vol. 3, no. 4, pp. 159-168, 2017. doi: https://doi.org/10. 20469/ijtes.3.40004-4

[12] M. Nenad and A. Zoran, "Multi-criteria decision making methods: Comparative analysis of promethee and vikor," in XVII International Scientific Conference on Industrial Systems, Novi Sad, Serbia, 2017.

[13] T. H. K. Kang and J. Ki Hong, "Detailed analytical methods for simulation of concrete, target resistance against F-4D jet impact," Journal of Advances in Technology and Engineering Studies, vol. 4, no. 1, pp. 1-8, 2018. doi: https://doi.org/10. 20474/jater-4.1.4

[14] S. Cherdchoongam and V. Rungreunganun, "An application of analytical hierarchy process for ranking factors affecting of thai natural rubber Ribbed Smoked Sheets no.3 (RSS3) price," International Journal of Technology and Engineering Studies, vol. 1, no. 2, pp. 42-47, 2015. doi: https://doi.org/10.20469/ijtes.40002-2

[15] H. Polatidis, D. A. Haralambopoulos, G. Munda, and R. Vreeker, "Selecting an appropriate multi-criteria decision analysis technique for renewable energy planning," Energy Sources, vol. 1, no. 2, pp. 181-193, 2006. doi: https://doi.org/10. $1080 / 009083190881607$

[16] H. A. A. Ribeiro, Evaluation and selection of innovation projects. New York, NY: Springer, 2015.

[17] T. L. Saaty, "Decision making with the analytic hierarchy process," International Journal of Services Sciences, vol. 1, no. 1, pp. 83-98, 2008. doi: https://doi.org/10.1504/IJSSci.2008.01759

[18] T. L. Saaty, Theory and applications of the analytic network process: decision making with benefits, opportunities, costs, and risks. Pittsburgh, PA: RWS Publications, 2005.

[19] B. B. Seels and R. C. Richey, "Instructional technology: The definition and domains of the field," Journal of Instructional Development, vol. 1, no. 1, pp. 5-8, 1994.

[20] H. Farman, H. Javed, B. Jan, J. Ahmad, S. Ali, F. Khalil, and M. Khan, "Analytical network process based optimum cluster head selection in wireless sensor network," PLOS ONE, vol. 12, no. 7, pp. 34-57, 2017. doi: https://doi.org/10.1371/ journal.pone. 0180848 